\title{
Long-term clinical outcomes and radiological findings and their correlation with each other after standard open discectomy for lumbar disc herniation
}

\author{
*II-Nam Son, MD, Young-Hoon Kim, MD, and Kee-Yong Ha, MD \\ Department of Orthopaedic Surgery, Seoul St. Mary's Hospital, College of Medicine, The Catholic University of Korea,
} Seoul, Korea

\begin{abstract}
OBJECT This retrospective study was designed to evaluate the clinical outcomes and radiological findings after open lumbar discectomy (OLD) in patients who were followed up for 10 years or longer.

METHODS The authors classified 79 patients who had a mean age ( \pm SD) of $53.6 \pm 13.6$ years (range 30-78 years) into 4 groups according to the length of their follow-up. Patients in Group 1 were followed up for 10-14 years, in Group 2 for 15-19 years, in Group 3 for 20-24 years, and in Group 4 for more than 25 years. In all of these patients, the clinical outcomes were assessed by using patients' self-reported scores on visual analog scales (VASs) measuring back and leg pain and by using scores from the Oswestry Disability Index (ODI). In addition, 10 radiological parameters suggesting degenerative changes or instability at the operated segment were recorded at various time points and used to calculate a numeric radiological finding (NRF) score by rating a presence for each finding of spinal degeneration or instability as 1. RESULTS The authors observed that OLD decreased pain and disability scores in all groups. Numeric radiological findings were highest in Group 4, and a significant correlation was detected between NRFs and VAS scores of back pain ( $p$ $=0.039$ ). In this cohort, the reoperation rate was $13.9 \%$ during a mean follow-up period of 15.3 years. Clinical outcomes tended to be most favorable in Group 1, representing patients who had OLD most recently, and they tended to deteriorate in the other 3 groups, indicating some worsening of outcomes over time. Degeneration of the spine at the operated level measured with radiographic methods tended to increase over time, but some stabilization was observed. Although spinal degeneration was stable, clinical outcomes deteriorated over time.
\end{abstract}

CONCLUSIONS This cross-sectional assessment of a retrospective cohort indicates that outcomes after OLD deteriorate over time. Increased back pain indicated a worsening of clinical outcomes, and this worsening was correlated with radiological findings of degeneration at the operated segment.

http://thejns.org/doi/abs/10.3171/2014.10.SPINE131126

KEY WORDS lumbar disc herniation; open discectomy; long-term follow-up; spinal disorders

$\mathrm{T}$ HE notion that lumbar disc herniation (LDH) causes neural compression was first proposed by Mixter and Barr. ${ }^{14}$ For more than 70 years, standard open lumbar discectomy (OLD) has been used to manage LDH in patients who have persistent symptoms of the condition that do not improve with a conservative treatment. ${ }^{20}$ Although minimally invasive surgery has recently gained attention, most spine surgeons still prefer standard OLD. Moreover, there has been no evidence suggesting that newer discectomy techniques are superior to open discectomy, and patients undergoing an OLD usually have satisfactory functional scores. ${ }^{9,22}$ However, variable long-term results and worsening of patients' conditions over time have been reported. ${ }^{9,17,18,22}$ In particular, patients may experience back pain after an OLD for an $\mathrm{LDH}^{3-5}$ Several studies have observed long-term postoperative changes of the lumbar spine in patients who had undergone discectomy for an $\mathrm{LDH} .{ }^{2,8,11,13,18,21,23}$ Yorimitsu et al. ${ }^{23}$ reported a $25 \%$ loss of the disc height 10 years after a discectomy, and Barth et al. ${ }^{2}$ reported a significant increase in endplate degeneration after discectomies. However, long-term follow-up studies of the relationship between these radiological findings and clinical outcomes are rare.

Therefore, the purpose of the present study was to analyze the clinical outcomes and radiological findings in patients who had been followed up for at least 10 years after

ABBREVIATIONS ADHR = anterior disc height ratio; LDH = lumbar disc herniation; NRF = numeric radiological finding; ODI = Oswestry Disability Index; OLD = open lumbar discectomy; PDHR = posterior disc height ratio; VAS = visual analog scale.

SUBMITTED December 9, 2013. ACCEPTED October 16, 2014.

INCLUDE WHEN CITING Published online November 28, 2014; DOI: 10.3171/2014.10.SPINE131126.

DISCLOSURE This study was partly supported by the AOSpine Foundation.

* Drs. Son and Kim contributed equally to this work. 
an OLD. We also investigated the possible associations of these findings with the patients' self-reported levels of pain and long-term functional outcomes. Additionally, we investigated the reoperation rates at $\geq 10$ years after an OLD.

\section{Methods \\ Study Design}

This study was performed with the approval of our hospital's institutional review board. Patients who had undergone OLD for a 1-level LDH at our institution were selected. Cross-sectional analysis of clinical and radiological observations was used to investigate the long-term outcomes of OLDs. To reduce any bias due to selection from a heterogeneous patient population, the following inclusion criteria were used: 1) a single-level OLD, at either L4-5 or L5-S1, 2) a length of follow-up of 10 years or longer, and 3) the last follow-up examination between January 2010 and December 2010. Patients who had a previous lumbar surgery, a reoperation within 10 years, or age greater than 60 years at the time of the initial OLD were excluded. Seventy-nine patients who met the inclusion criteria and for whom clinical and radiological data were available were included in this study.

The patients' records were reviewed retrospectively. Forty men and 39 women with a mean age $( \pm$ SD) of 53.6 \pm 13.6 years (range $30-78$ years) were included. The mean age at surgery was $37.6 \pm 12.8$ years (range 18-59 years). The mean length of follow-ups was $15.3 \pm 6.1$ years (range 10-32 years). Fifty-eight patients underwent an L4-5 OLD and 21 patients an L5-S1 OLD. The types of herniation and the number of cases for each type were as follows: 17 protrusions, 11 extrusions, and 28 sequestrations. The remaining 23 cases could not be classified. The 79 patients were classified into 4 groups according to the length of the follow-up. Group 1 had 46 patients who had been followed up for 10-14 years, Group 2 had 19 patients followed up for 15-19 years, Group 3 had 7 patients followed up for 20-24 years, and Group 4 had 7 patients followed up for $>25$ years.

\section{Clinical Outcomes}

Clinical outcomes before and after surgery were assessed by having patients score any pain in their legs or lower back on visual analog scales (VASs) and their level of pain-related disability on the Oswestry Disability Index (ODI). Preoperative clinical data were not available for 16 patients (20\%) who mainly belonged to Groups 3 and 4.

\section{Radiological Assessments}

Radiological assessments were performed at various times in the follow-up periods indicated for each group and at the last follow-up for 11 patients who underwent a reoperation. The assessments were performed with plain radiographs, including anteroposterior and neutral radiographs, and dynamic standing lateral radiographs.

Local kyphosis at the operated segment was determined by measuring the angle of the operated segment, that is, the angle between the lower endplate of the upper vertebral body and the upper endplate of the lower vertebral body. Lumbar lordosis was defined as the angle between the upper endplate of L-1 and the lower endplate of L-5. The anterior disc height ratio (ADHR) was defined as the anterior disc height of the operated segment (b) divided by the anterior disc height of the upper segment (a) (that is, $\mathrm{ADHR}=\mathrm{b} / \mathrm{a})$. The posterior disc height ratio (PDHR) was defined as the posterior disc height of the operated segment (d) divided by the posterior disc height of the upper segment (c) (that is, PDHR $=\mathrm{d} / \mathrm{c}$ ). An intradiscal vacuum phenomenon suggesting spinal instability on dynamic radiographs (Knutsson's sign), osteophytes suggesting instability of the segment (traction spur), osteophytes related to repetitive compressive loads (claw spur), and upper and lower endplate sclerosis were also recorded. A listhesis of $>2 \mathrm{~mm}$ was recorded as 1 of 3 instability findings, that is, anterior listhesis, retrolisthesis, or lateral listhesis (Fig. 1). To measure findings of spinal degeneration or instability on radiographs, a numeric radiological finding (NRF) scoring system was used by assigning a score of 1 for occurrence of each of 10 radiological findings.

Lateral wedging of the operated segment was recorded as an anteroposterior angulation at the operated segment, and motion of the operated segment, which was defined as the segmental angular motion (that is, motion of the operated segment $=b-a$ ) on flexion and extension on standing lateral radiographs, was also recorded (Fig. 2).

\section{Statistical Analysis}

The statistical analyses were performed with SPSS (version 17.0). Paired t-tests were used to analyze the differences in VAS scores of back and leg pain and in ODI scores between the preoperative and last follow-up examinations. Mann-Whitney U-tests and Fisher exact tests with the appropriate Bonferroni corrections for multiple comparisons were used to analyze the differences among the groups. A linear regression was used to analyze correlations of clinical outcomes with the NRFs indication degeneration or instability at the operated segment. A Kruskal-Wallis test was used to analyze the differences between the LDH type and disc height ratio. A survivorship analysis was used to estimate rates of nonreoperation. A difference was considered to be statistically significant at a $\mathrm{p}<0.05$. Mean results are presented $\pm \mathrm{SD}$.

\section{Results}

\section{Clinical Outcomes}

At the last follow-up, the mean VAS scores for back pain in Groups $1-4$ were $2.7 \pm 2.4,3.6 \pm 2.1,2.8 \pm 0.9$, and $5.5 \pm 4.5$, respectively, indicating significant improvement compared with the preoperative scores (the mean preoperative VAS score for back pain was $8.5 \pm 2.4)(\mathrm{p}<0.01)$. The mean VAS scores for leg pain in Groups 1-4 were 2.5 $\pm 2.5,3.3 \pm 2.1,2.7 \pm 2.3$, and $2.7 \pm 2.3$, respectively, also indicating a significant postoperative improvement (the mean preoperative VAS score for leg pain was $8.4 \pm 1.9$ ) $(\mathrm{p}<0.01)$. The postoperative ODI scores were also significantly improved in all groups; the mean scores for Groups $1-4$ were $17.9 \pm 13.4,19.8 \pm 12.5,18.5 \pm 17.2$, and $21.5 \pm$ 14.3 , respectively, and the mean preoperative ODI score was $65.8 \pm 23.5(\mathrm{p}<0.01)$. No statistically significant differences in these 3 clinical outcomes were detected among the 4 groups. 

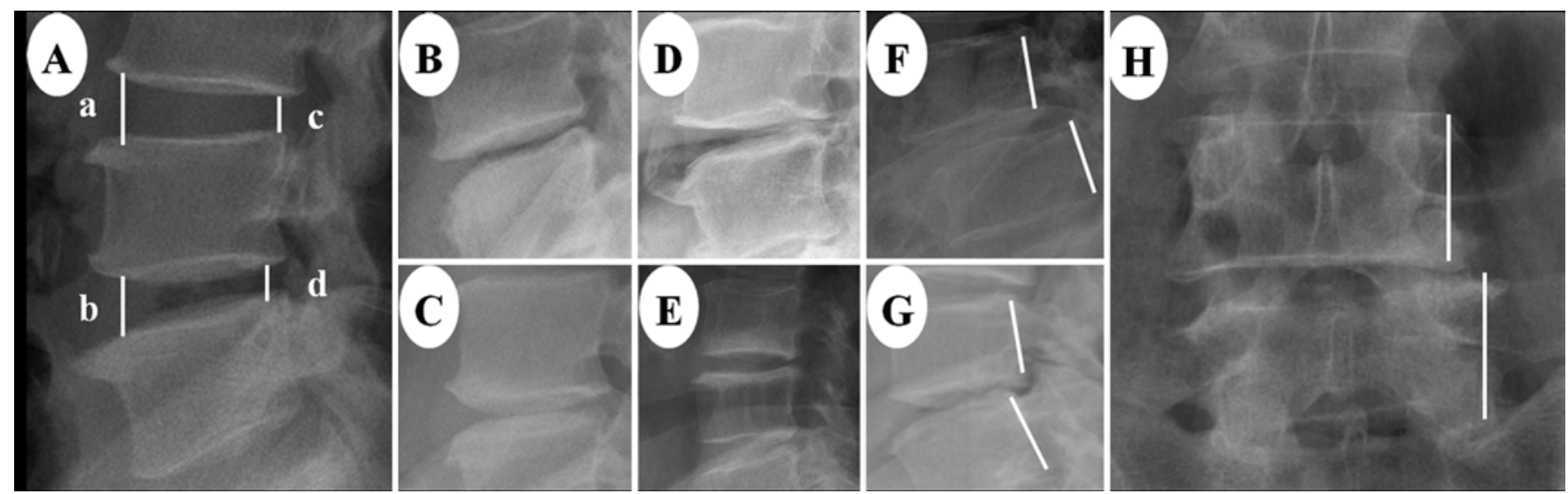

FIG. 1. Radiological findings used for assessment of degeneration or instability at the operated segment. A: The ADHR was defined as the anterior disc height of the operated segment (b) divided by the anterior disc height of the upper segment (a) (ADHR $=b / a)$. The PDHR was defined as the posterior disc height of the operated segment (d) divided by the posterior disc height of the upper segment $(\mathrm{c})(\mathrm{PDHR}=\mathrm{d} / \mathrm{c})$. Knutsson's sign $(\mathrm{B})$, traction spur $(C)$, claw spur $(\mathrm{D})$, and upper and lower endplate sclerosis $(\mathrm{E})$. A listhesis of $>2 \mathrm{~mm}$ was recorded as one of 3 instability findings: anterior listhesis $(\mathrm{F})$, retrolisthesis $(\mathrm{G})$, or lateral listhesis $(\mathrm{H})$.

\section{Radiological Findings Indicating Spinal Degeneration}

Local Kyphosis and Lumbar Lordosis

The mean local kyphosis angles at the operated level in Groups $1-4$ were $7.4^{\circ} \pm 4.0^{\circ}, 7.4^{\circ} \pm 4.0^{\circ}, 5.9^{\circ} \pm 3.4^{\circ}$, and $6.3^{\circ} \pm 3.1^{\circ}$, respectively, suggesting a decrease in local kyphosis in Groups 3 and 4 relative to the other 2 groups. The mean lumbar lordosis angles in Groups 1-4 were $47.2^{\circ} \pm 30.2^{\circ}, 47.2^{\circ} \pm 8.7^{\circ}, 41.0^{\circ} \pm 18.8^{\circ}$, and $39.7 \pm 11.9^{\circ}$, respectively. The lumbar lordosis also showed a decrease in Groups 3 and 4. However, no significant differences in lumbar lordosis were observed among the 4 groups.

\section{Disc Height}

The ADHR tended to decrease in Groups 3 and 4, indicated by mean ADHR values of $0.80 \pm 0.23,0.82 \pm 0.23$, $0.71 \pm 0.18$, and $0.72 \pm 0.18$ for Groups $1-4$, respectively. The mean PDHR values were $0.74 \pm 0.16,0.75 \pm 0.13,0.63$ \pm 0.14 , and $0.59 \pm 0.18$, respectively. No statistically signif- icant differences in the 2 disc height ratios were observed among the 4 groups.

\section{Anteroposterior Angulation and Motion at the Operated Segment}

The mean angles measured by anteroposterior angulation at the operated segment in Groups $1-4$ were $1.17^{\circ}$ $\pm 1.05^{\circ}, 1.08^{\circ} \pm 1.43^{\circ}, 2.48^{\circ} \pm 2.33^{\circ}$, and $2.65^{\circ} \pm 4.19^{\circ}$, respectively, suggesting an increase of this variable in Groups 3 and 4. However, the differences in the anteroposterior angles among the groups were not statistically significant. The means for the motions of the operated segment in Groups $1-4$ were $4.84^{\circ} \pm 2.76^{\circ}, 4.46^{\circ} \pm 3.09^{\circ}$, $3.69^{\circ} \pm 1.89^{\circ}$, and $3.91^{\circ} \pm 3.36^{\circ}$, respectively, suggesting a trend of gradual decrease in the motion with an increase in the length of follow-up. However, these differences among the groups were again not statistically significant.

\section{Endplate Sclerosis}

Upper endplate sclerosis was noted in 35 of the 46
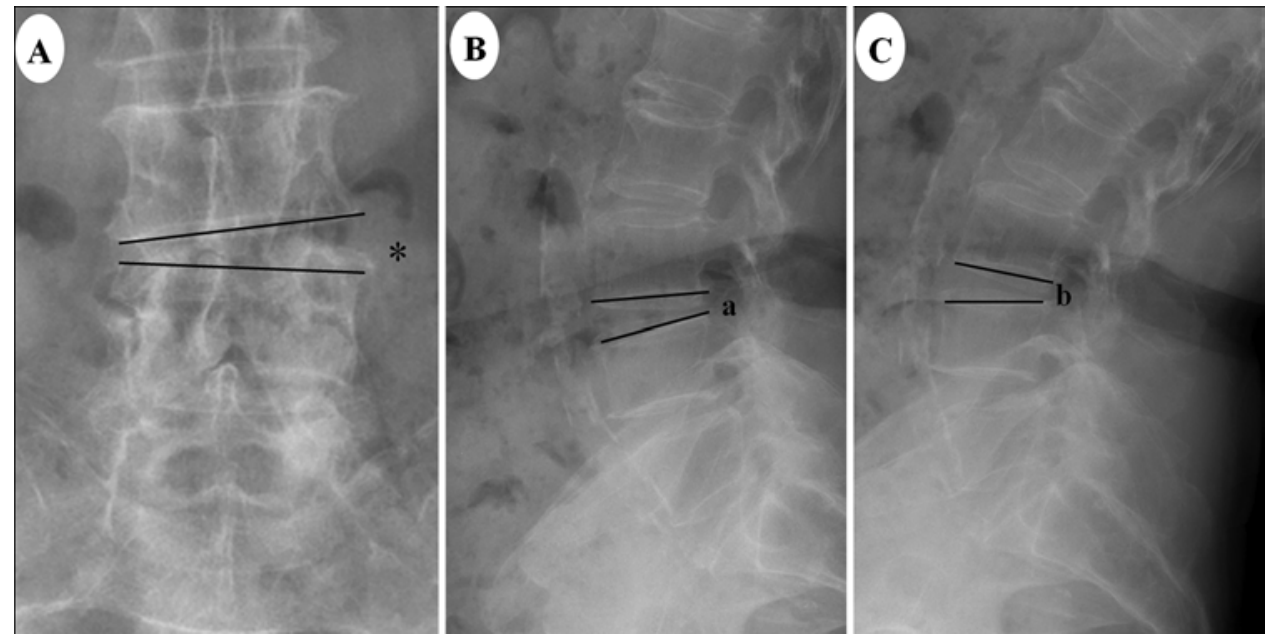

FIG. 2. Lateral wedging of the intervertebral disc and motion of the operated segment. A: Lateral wedging of the intervertebral disc was determined by anteroposterior angulation at the operated segment (shown by asterisk) on anteroposterior radiographs. B and C: Motion of the operated segment (MOS), which was defined as the segmental angular motion (MOS $=b-a)$ on flexion and extension was measured on dynamic lateral radiographs. 
patients (76\%) in Group 1, in 12 of 19 patients $(63 \%)$ in Group 2, in 6 of 7 patients (86\%) in Group 3, and in 3 of 7 patients (43\%) in Group 4. Lower endplate sclerosis was noted in 32 of 46 patients (70\%) in Group 1, in 10 of 19 patients (53\%) in Group 2, in 5 of 7 patients (71\%) in Group 3 , and in 7 of 7 patients (100\%) in Group 4. Sclerosis in both endplates was observed in 28 of 46 patients $(61 \%)$ in Group 1, in 10 of 19 patients (53\%) in Group 2, in 5 of 7 patients (71\%) in Group 3, and in 7 of 7 patients (100\%) in Group 4 . A statistically significant difference in the presence of sclerosis in both endplates was detected between Groups 1 and $4(\mathrm{p}=0.03)$.

\section{Claw Spur}

Claw spurs were observed in 1 of the 46 patients (2\%) in Group 1, in none of the patients in Group $2(0 \%)$, in 2 of 7 patients (29\%) in Group 3, and in 3 of 7 patients (43\%) in Group 4. These rates suggested an increased prevalence of claw spurs among patients of Groups 3 and 4, and a statistically significant difference in claw spur prevalence was detected between Groups 1 and $4(\mathrm{p}=0.009)$.

\section{Radiological Findings Indicating Spinal Instability Knutsson's Sign}

In this study, Knutsson's sign was used as an indicator for spinal instability; Knutsson's sign was noted in 18 of the 46 patients (39\%) in Group 1, in 8 of 19 patients (42\%) in Group 2, in 6 of 7 patients (86\%) in Group 3, and in 4 of 7 patients (57\%) in Group 4. A statistically significant difference in the prevalence of Knutsson's sign was detected between Groups 1 and $3(\mathrm{p}=0.046)$.

\section{Traction Spur}

A traction spur was observed in 32 of the 46 patients $(70 \%)$ in Group 1, in 6 of 19 patients (32\%) in Group 2, in 3 of 7 patients (42\%) in Group 3, and in 2 of 7 patients (29\%) in Group 4. No significant differences in the prevalence of traction spurs were observed among the 4 groups.

\section{Listhesis}

No anterior listhesis was observed in any of the patients. A retrolisthesis was noted in 4 of 46 patients $(9 \%)$ in Group 1, in 1 of 19 patients (5\%) in Group 2, in 0 of 7 patients $(0 \%)$ in Group 3, and in 2 of 7 patients $(29 \%)$ in Group 4. No significant differences among the 4 groups were detected. Lateral listhesis was seen in 3 of 46 patients (6.5\%) in Group 1, in 3 of 19 patients (16\%) in Group 2, in 1 of 7 patients (14\%) in Group 3, and in 1 of 7 patients $(14 \%)$ in Group 4. No significant differences in the prevalence of lateral listhesis were detected between any of the 4 groups.

\section{Numeric Radiological Findings}

The mean NRF scores for Groups $1-4$ were $3.7 \pm 1.9$, $4.3 \pm 1.6,5.3 \pm 0.9$, and $5.6 \pm 1.1$, respectively, indicating an increase in the NRF score in Group 4, and a significant difference between Groups 1 and 4 was detected $(\mathrm{p}=0.03)$. The most common NRF among the patients was a decreased PDHR. The patients were classified into 2 groups according to the type of NRF: one group contained those patients who had 5 findings of spinal degeneration (that is, decreased ADHR or PDHR, claw spur, and upper and lower endplate sclerosis), and the other group comprised patients who had 5 findings indicating spinal instability (that is, Knutsson's sign, traction spur, and anterior listhesis, retrolisthesis, or lateral listhesis). The mean scores for spinal degeneration in Groups $1-4$ were $2.7 \pm 1.3,3.3 \pm$ $0.9,3.9 \pm 0.4$, and $4.3 \pm 0.5$, respectively, suggesting that spinal degeneration increased with time. Statistically significant differences in spinal degeneration were detected between Groups 1 and 2, 1 and 3, as well as between 1 and $4(\mathrm{p}=0.045, \mathrm{p}=0.022$, and $\mathrm{p}<0.001$, respectively). The mean scores for spinal instability in Groups 1-4 were 1.0 $\pm 0.9,0.9 \pm 1.1,1.4 \pm 0.9$, and $1.3 \pm 1.1$, respectively, and no significant differences among the groups were detected.

\section{Correlation of NRFs With Clinical Outcomes}

The relationships between NRFs and each of the 3 clinical outcomes (the ODI scores and the VAS scores of pain in the back and leg) were analyzed by linear regression. A significant correlation was observed between NRFs and VAS scores for back pain $(\mathrm{p}=0.039)$. That is, back pain significantly increased with the number of radiological findings (Fig. 3). No significant correlations of NRFs with VAS leg pain scores or with ODI scores were observed ( $p$ $=0.318$ and $\mathrm{p}=0.093$, respectively).

\section{Relationship Between the Type of Herniation and the Disc Height Ratio}

The mean ADHR at the last follow-up radiographic examination was $0.76 \pm 0.27$ for protrusion, $0.88 \pm 0.20$ for extrusion, and $0.78 \pm 0.20$ for sequestration. No significant correlation between the type of herniation and the ADHR was observed $(\mathrm{p}=0.362)$. The mean PDHR was $0.74 \pm$ 0.19 for protrusion, $0.76 \pm 0.14$ for extrusion, and $0.76 \pm$ 0.14 for sequestration, and again no significant correlation between the type of herniation and the PDHR was detected $(p=0.972)$ (Fig. 4).

\section{Nonreoperation Rate and Outcomes of Revision}

The reoperation rate was $13.9 \%$ (11 of 79 patients) in this cohort. The 11 patients $(6$ men and 5 women with a mean age of $51.2 \pm 13.9$ years) had all undergone revision surgery. Four patients underwent fusion for degenerative stenosis and 7 underwent OLD reoperation for a recurring LDH. All revision discectomies were performed at the same level as the first operation, and spinal fusion included the previously operated level. The mean age at the first operation of the patients who underwent a reoperation was $31.3 \pm 14$ years (range $18-59$ years) and their mean follow-up duration was $18.9 \pm 8.6$ years (range 10-31 years). Patients who underwent fusion surgery were older than patients who underwent standard repeat discectomy. The average age of the patients who underwent OLD reoperation was $42.7 \pm 8.9$ years (range $30-51$ years), and the average age of the patients who underwent fusion was 66.0 \pm 5.0 years (range 62-72 years). Three patients in Group 1 had a recurring $\mathrm{LDH}$, and 1 underwent fusion for a degenerative stenosis; 2 patients in Group 2 underwent OLD reoperation for a recurring $\mathrm{LDH}$, and 1 underwent fusion for a degenerative stenosis; 1 patient in Group 3 underwent fusion for a degenerative stenosis; and 2 patients in Group 
- Back pain VAS $=\mathrm{NRF}$

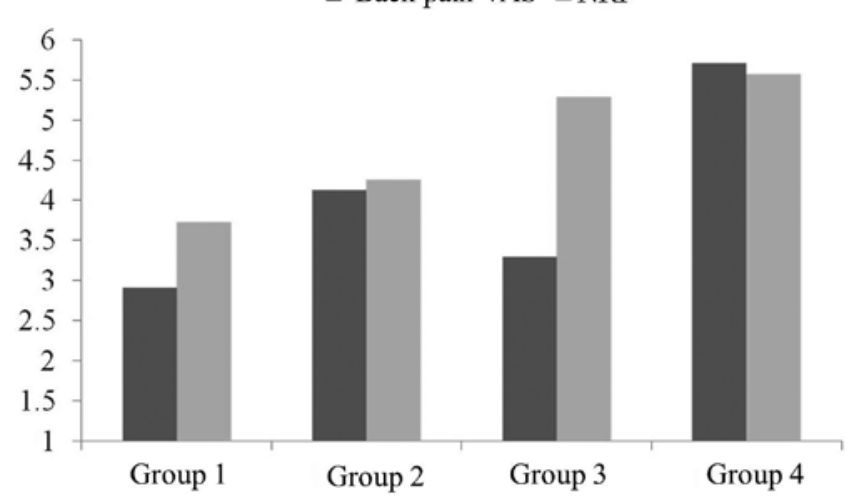

FIG. 3. Correlation of VAS back pain scores with NRFs. VAS back pain scores significantly increased with an increase in the number of NRFs. Linear regression analysis indicated that the correlation between NRFs and back pain scores was statistically significant $(p=0.039)$.

4 underwent OLD reoperation for a recurring $\mathrm{LDH}$, and 1 underwent fusion for a degenerative stenosis.

A survivorship analysis was performed to assess the rate of nonreoperation for all patients, taking into account patients lost to follow-up. The estimated rate of nonreoperation was $80.9 \%$ at 20 years and $57.2 \%$ after 30 years (Fig. 5). These findings suggested that $19.1 \%$ of all patients underwent or would undergo a reoperation within 20 years and that $42.8 \%$ underwent or would undergo a reoperation at or after 30 years.

\section{Discussion}

Open lumbar discectomy has been used as a gold standard for surgical interventions to treat limited refractory LDH. Although many authors have suggested that OLD causes spondylosis, which contributes to mechanical back pain, ${ }^{1,2,10,11,23}$ several studies have failed to identify a correlation between the clinical and radiological outcomes of an OLD. ${ }^{8,11,18,21}$ Other authors have proposed that back pain after an OLD is associated with disc degeneration as a result of disc space narrowing or endplate sclerosis. ${ }^{13,15,16}$ Because it is difficult to establish whether specific radiological findings are associated with certain clinical outcomes, we wanted to determine which radiological findings may be associated with common clinical outcomes of an OLD. Moreover, to focus on the long-term effects of OLD for LDH on radiological and clinical outcomes, only patients treated with OLD who had been followed up for 10 years or longer were included in this study.

The NRFs consisted of 5 findings of spinal degeneration and 5 findings of spinal instability. ${ }^{10,12}$ The VAS scores for back or leg pain and the ODI scores were lower in Group 1 and increased slightly over time as indicated by some gradual increases in the scores for the other 3 groups containing patients with a longer follow-up, and NRFs also increased over time. It has been suggested that an increase in NRF scores in patients makes it more likely that clinical outcomes for these patients are the result of spinal deterioration. A significant correlation was indeed observed between the VAS back pain scores and the NRFs $(p=0.039)$. However, no correlations were detected of the VAS leg pain scores with the NRFs or with ODI scores

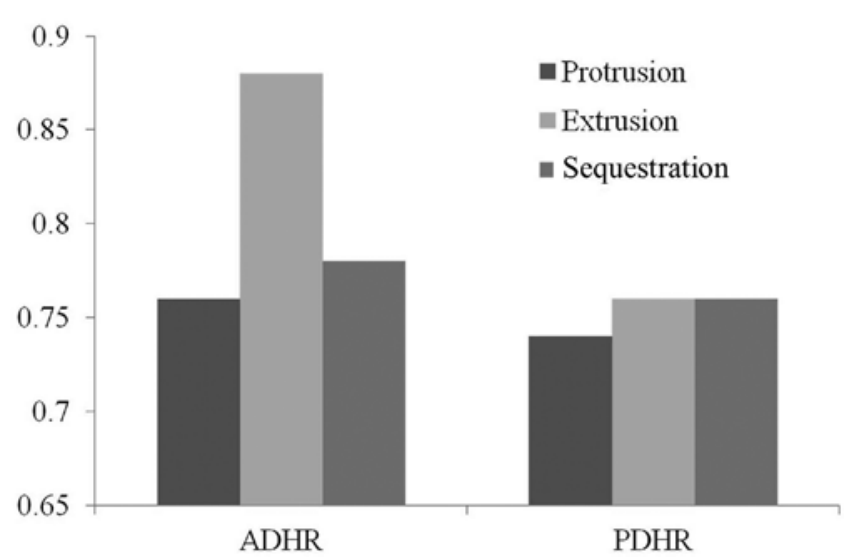

FIG. 4. Relationship between the type of herniation and the disc height ratios. No statistically significant correlation was detected between the type of herniation and the size of an ADHR or a PDHR ( $p=0.362$ and $p$ $=0.972$, respectively).

( $\mathrm{p}=0.318$ and $\mathrm{p}=0.093$, respectively). We conclude that the NRFs were significantly associated with back pain but not with leg pain or functional disability. As back pain is not solely related to 1-level degeneration, for example, at the operated segments, we could not firmly conclude that the radiographic changes at the operated segment are significantly correlated with the back pain. However, radiographic changes suggesting degeneration and instability at the operated level were associated with an increased incidence of back pain in this cross-sectional examination of a long-term postoperative cohort.

The present study showed that after an OLD, with older patients included, the VAS scores for leg pain improved more than those for back pain (except in Group 4). However, it remains unclear whether worsening VAS scores for back pain were due to spinal degeneration progressing with the natural aging process or were the result of accelerated degeneration after an OLD.

Several studies have reported rates of reoperation of 6\%-17\% during long-term follow-up periods. ${ }^{6,7,23}$ In the present study, the reoperation rate was $13.4 \%$ during a mean follow-up period of 15.3 years. The rate of nonreoperation was measured with the survivorship analysis and was estimated to be $80.9 \%$ at 20 years and $57.2 \%$ after 30 years. This result suggests that $19.1 \%$ of all patients did or will undergo reoperation as a result of OLD within

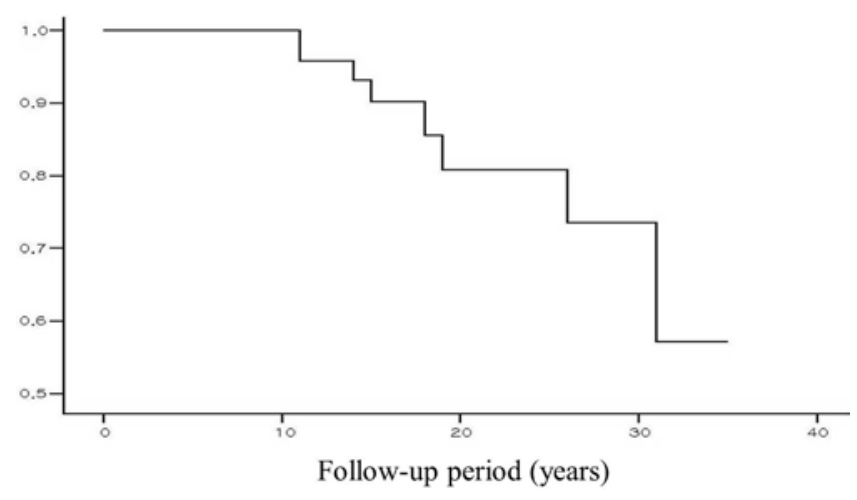

FIG. 5. Rates of nonreoperation. The estimated rates of nonreoperation after an OLD were $80.9 \%$ within 20 years and $57.2 \%$ after 30 years. 
20 years and that $42.8 \%$ did or will undergo reoperation within or after 30 years. In this study, 4 patients had undergone spinal fusion for a degenerative stenosis. Patients undergoing a fusion were older than patients undergoing an OLD reoperation. It is thought that radiological observations of stabilization indicating osteophyte development and disc space narrowing are signs of spinal stenosis. The results in this study suggest that if the follow-up period is sufficiently long, the stabilization phase or degeneration due to aging both contribute to degenerative stenosis.

There are 3 types of LDH caused by a protruded, extruded, or sequestrated disc. ${ }^{19}$ To the best of our knowledge, no reports exist that have correlated the type of $\mathrm{LDH}$ with a collapse of the disc height. We analyzed the magnitude of the change in the disc height ratio depending on the type of LDH. However, at the final follow-up, no significant differences were observed in disc height ratios between the 3 different types of LDH.

A limitation of this study was that it represents a crosssectional assessment with retrospectively collected data from a patient cohort without a control group. Although data from serial radiological examinations were available for many patients, the radiological findings were mainly obtained at the last follow-up. Therefore, the present study could not present any significant information in terms of the natural course of LDH. However, the long follow-up period over more than 10 years postoperatively enabled collection of long-term outcome data after OLD for an LHD. Another limitation is the small number of patients enrolled over time. Enrollment of a larger number of patients would have yielded more accurate results.

\section{Conclusions}

The observations in this cross-sectional study indicate that clinical outcomes after OLD tend to deteriorate over time. Back pain was significantly associated with worsening of the clinical outcomes and was also correlated with radiological changes.

\section{References}

1. Balderston RA, Gilyard GG, Jones AA, Wiesel SW, Spengler DM, Bigos SJ, et al: The treatment of lumbar disc herniation: simple fragment excision versus disc space curettage. J Spinal Disord 4:22-25, 1991

2. Barth M, Diepers M, Weiss C, Thomé C: Two-year outcome after lumbar microdiscectomy versus microscopic sequestrectomy: part 2: radiographic evaluation and correlation with clinical outcome. Spine (Phila Pa 1976) 33:273-279, 2008

3. Carragee EJ, Han MY, Yang B, Kim DH, Kraemer H, Billys J: Activity restrictions after posterior lumbar discectomy. A prospective study of outcomes in 152 cases with no postoperative restrictions. Spine (Phila Pa 1976) 24:2346-2351, 1999

4. Carragee EJ, Spinnickie AO, Alamin TF, Paragioudakis S: A prospective controlled study of limited versus subtotal posterior discectomy: short-term outcomes in patients with herniated lumbar intervertebral discs and large posterior anular defect. Spine (Phila Pa 1976) 31:653-657, 2006

5. Caspar W, Campbell B, Barbier DD, Kretschmmer R, Gotfried Y: The Caspar microsurgical discectomy and comparison with a conventional standard lumbar disc procedure. Neurosurgery 28:78-87, 1991

6. Davis RA: A long-term outcome analysis of 984 surgically treated herniated lumbar discs. J Neurosurg 80:415-421, 1994
7. Dvorak J, Gauchat MH, Valach L: The outcome of surgery for lumbar disc herniation. I. A 4-17 years' follow-up with emphasis on somatic aspects. Spine (Phila Pa 1976) 13:1418-1422, 1988

8. Hanley EN Jr, Shapiro DE: The development of low-back pain after excision of a lumbar disc. J Bone Joint Surg Am 71:719-721, 1989

9. Hoffman RM, Wheeler KJ, Deyo RA: Surgery for herniated lumbar discs: a literature synthesis. J Gen Intern Med 8:487-496, 1993

10. Kirkaldy-Willis WH, Farfan HF: Instability of the lumbar spine. Clin Orthop Relat Res (165):110-123, 1982

11. Loupasis GA, Stamos K, Katonis PG, Sapkas G, Korres DS, Hartofilakidis G: Seven- to 20-year outcome of lumbar discectomy. Spine (Phila Pa 1976) 24:2313-2317, 1999

12. Macnab I: The traction spur. An indicator of segmental instability. J Bone Joint Surg Am 53:663-670, 1971

13. Mariconda M, Galasso O, Attingenti P, Federico G, Milano $\mathrm{C}$ : Frequency and clinical meaning of long-term degenerative changes after lumbar discectomy visualized on imaging tests. Eur Spine J 19:136-143, 2010

14. Mixter WJ, Barr JS: Rupture of the intervertebral disc with involvement of the spinal canal. N Engl J Med 211:210-215, 1934

15. Peterson CK, Bolton JE, Wood AR: A cross-sectional study correlating lumbar spine degeneration with disability and pain. Spine (Phila Pa 1976) 25:218-223, 2000

16. Pye SR, Reid DM, Smith R, Adams JE, Nelson K, Silman AJ, et al: Radiographic features of lumbar disc degeneration and self-reported back pain. J Rheumatol 31:753-758, 2004

17. Salenius P, Laurent LE: Results of operative treatment of lumbar disc herniation. A survey of 886 patients. Acta Orthop Scand 48:630-634, 1977

18. Saruhashi Y, Mori K, Katsuura A, Takahashi S, Matsusue Y, Hukuda S: Evaluation of standard nucleotomy for lumbar disc herniation using the Love method: results of follow-up studies after more than 10 years. Eur Spine J 13:626-630, 2004

19. Semmes RE: Diagnosis of ruptured intervertebral disc without contrast myelography and comment upon recent experience with modified hemilaminectomy for their removal. Yale J Biol Med 11:433-435, 1939

20. Spengler DM, Ouellette EA, Battié M, Zeh J: Elective discectomy for herniation of a lumbar disc. Additional experience with an objective method. J Bone Joint Surg Am 72:230237,1990

21. Takeshima T, Kambara K, Miyata S, Ueda Y, Tamai S: Clinical and radiographic evaluation of disc excision for lumbar disc herniation with and without posterolateral fusion. Spine (Phila Pa 1976) 25:450-456, 2000

22. Weber H: Lumbar disc herniation. A controlled, prospective study with ten years of observation. Spine (Phila Pa 1976) 8:131-140, 1983

23. Yorimitsu E, Chiba K, Toyama Y, Hirabayashi K: Long-term outcomes of standard discectomy for lumbar disc herniation: a follow-up study of more than 10 years. Spine (Phila Pa 1976) 26:652-657, 2001

\section{Author Contributions}

Conception and design: Ha, Kim. Acquisition of data: Son. Analysis and interpretation of data: Son. Drafting the article: Son. Critically revising the article: Kim. Reviewed submitted version of manuscript: Ha. Statistical analysis: Kim.

\section{Correspondence}

Kee-Yong Ha, Department of Orthopaedic Surgery, Seoul St. Mary's Hospital, The Catholic University of Korea, College of Medicine, 505 Banpo-Dong, Seocho-Gu, Seoul 137-701, Korea. email: kyh@catholic.ac.kr. 